\title{
Signal transducer and activator of transcription 1 (STAT1) gain- of-function mutations and disseminated coccidioidomycosis and histoplasmosis
}

\author{
Elizabeth P. Sampaio, MD, PhD ${ }^{a, b}$, Amy P. Hsu, BA ${ }^{a}$, Joseph Pechacek, BA ${ }^{a}$, Hannelore I. \\ Bax, MD ${ }^{a, c}$, Dalton L. Dias, BA ${ }^{a}$, Michelle L. Paulson, MDd, Prabha Chandrasekaran, PhD ${ }^{a}$, \\ Lindsey B. Rosen, BS ${ }^{a}$, Daniel S. Carvalho, PhDa,b, Li Ding, MD ${ }^{a}$, Donald C. Vinh, MD $^{\mathrm{e}}$, \\ Sarah K. Browne, MDa ${ }^{a}$, Shrimati Datta, PhD $^{f}$, Joshua D. Milner, MD $^{f}$, Douglas B. Kuhns, \\ PhD' $^{g}$, Debra A. Long Priel, BS ${ }^{g}$, Mohammed A. Sadat, MD ${ }^{h}$, Michael Shiloh, MD, PhD', \\ Brendan De Marco, MDi, Michael Alvares, MDj, Jason W. Gillman, MDi, Vivek Ramarathnam, \\ MD', Maite de la Morena, MDj, Liliana Bezrodnik, MD', Ileana Moreira, MD', Gulbu Uzel, \\ MD $^{\mathrm{a}}$, Daniel Johnson, MD', Christine Spalding, RN ${ }^{\mathrm{a}}$, Christa S. Zerbe, MDa, Henry Wiley, \\ MD $^{m}$, David E. Greenberg, MD', Susan E. Hoover, MD, PhD ${ }^{n}$, Sergio D. Rosenzweig, MD, \\ $\mathbf{P h D}^{\mathrm{h}, \mathrm{o}}$, John N. Galgiani, $\mathbf{M D}^{\mathrm{n}}$, and Steven M. Holland, $\mathbf{M D}^{\mathrm{a}}$ \\ athe Immunopathogenesis Section, Laboratory of Clinical Infectious Diseases, Laboratory of Host \\ Defenses, National Institutes of Health $(\mathrm{NIH})$, Bethesda \\ bthe Leprosy Laboratory, Oswaldo Cruz Institute, FIOCRUZ, Rio de Janeiro \\ cthe Department of Internal Medicine and Department of Medical Microbiology and Infectious \\ Diseases, Erasmus Medical Center, Rotterdam \\ ${ }^{d}$ the Clinical Research Directorate/CMRP SAIC-Frederick, NCI-Frederick, Frederick \\ ethe Division of Infectious Diseases, McGill University Health Centre, Montreal \\ the Allergic Inflammation Unit, Laboratory of Allergic Diseases Laboratory of Host Defenses, \\ National Institutes of Health (NIH), Bethesda \\ gthe Clinical Services Program, SAIC-Frederick, NCI-Frederick, Frederick \\ hthe Infectious Diseases Susceptibility Unit, Laboratory of Host Defenses, National Institutes of \\ Health (NIH), Bethesda \\ ithe Division of Infectious Diseases, University of Texas Southwestern Medical Center, Dallas \\ Ithe Division of Allergy and Immunology, University of Texas Southwestern Medical Center, \\ Dallas \\ kthe Immunology Unit, Pediatric Hospital R. Gutierrez, Buenos Aires \\ 'Comer Children's Hospital, University of Chicago

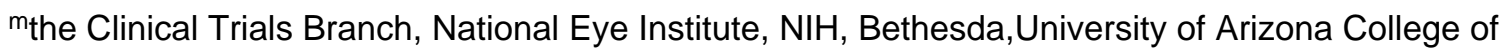 \\ Medicine, Tucson
}

Corresponding author: Steven M. Holland, MD, CRC B3-4141 MSC 1684, Bethesda, MD 20892-1684. h@nih.gov.

Disclosure of potential conflict of interest: D. C. Vinh has received research support from the Canadian Institutes of Health Research Post-doctoral Fellowship, CSL Behring Canada, and Astellas Canada; has consultant arrangements with CSL Behring Canada and Pfizer Canada; and has received payment for lectures from CSL Behring Canada and Sunovion/Sepracor. D. B. Kuhns and D. A. Long Priel have received grants from the National Cancer Institute/National Institutes of Health. V. Ramarathnam is employed by Private Practice ID. S. D. Rosenzweig receives royalties from UpToDate. The rest of the authors declare that they have no relevant conflicts of interest. 
nValley Fever Center for Excellence, University of Arizona College of Medicine, Tucson

othe Primary Immunodeficiency Clinic, National Institute of Allergy and Infectious Diseases

(NIAID), National Institutes of Health (NIH), Bethesda

\section{Abstract}

Background-Impaired signaling in the IFN- $\gamma /$ IL-12 pathway causes susceptibility to severe disseminated infections with mycobacteria and dimorphic yeasts. Dominant gain-of-function mutations in signal transducer and activator of transcription 1 (STATI) have been associated with chronic mucocutaneous candidiasis.

Objective-We sought to identify the molecular defect in patients with disseminated dimorphic yeast infections.

Methods-PBMCs, EBV-transformed B cells, and transfected U3A cell lines were studied for IFN- $\gamma / \mathrm{IL}-12$ pathway function. STAT1 was sequenced in probands and available relatives.

Interferon-induced STAT1 phosphorylation, transcriptional responses, protein-protein interactions, target gene activation, and function were investigated.

Results-We identified 5 patients with disseminated Coccidioides immitis or Histoplasma capsulatum with heterozygous missense mutations in the STAT1 coiled-coil or DNA-binding domains. These are dominant gain-of-function mutations causing enhanced STAT1 phosphorylation, delayed dephosphorylation, enhanced DNA binding and transactivation, and enhanced interaction with protein inhibitor of activated STAT1. The mutations caused enhanced IFN- $\gamma$-induced gene expression, but we found impaired responses to IFN- $\gamma$ restimulation.

Conclusion-Gain-of-function mutations in STAT1 predispose to invasive, severe, disseminated dimorphic yeast infections, likely through aberrant regulation of IFN- $\gamma$-mediated inflammation.

\section{Keywords}

Signal transducer and activator of transcription $1 ; \mathrm{IFN}-\gamma ;$ progressive multifocal leukoencephalopathy; Histoplasma capsulatum; Coccidioides immitis; thrush

The IFN- $\gamma / \mathrm{IL}-12$ signaling pathway controls extrapulmonary infections with bacteria, such as nontuberculous mycobacteria, BCG, Mycobacterium tuberculosis, and Salmonella species, ${ }^{1,2}$ as well as the dimorphic fungi Histoplasma capsulatum, ${ }^{3}$ Paracoccidioides brasiliensis, ${ }^{4}$ and Coccidioides immitis. ${ }^{5,6}$ Stimulation of IFN- $\gamma$ and IFN-a receptors leads to phosphorylation of signal transducer and activator of transcription 1 (STAT1), which homodimerizes and heterodimerizes before translocating to the nucleus, where interferoninduced genes are activated. ${ }^{7}$ Complete recessive mutations in $S T A T 1$ cause susceptibility to viral, mycobacterial, and bacterial infections, whereas heterozygous inhibitory STAT1 mutations cause mild disseminated BCG or nontuberculous mycobacterial infections. ${ }^{8-10}$ Recently, dominant gain-of-function mutations in $S T A T 1$ were described as causing chronic mucocutaneous candidiasis (CMC), impaired STAT1 dephosphorylation, and diminished numbers of IL-17-producing T cells. ${ }^{11,12}$

The regulation of STAT1 activity includes the suppressor of cytokine signaling and protein inhibitor of activated STAT (PIAS) families of proteins. ${ }^{13,14}$ Posttranslational modifications of STATs (acetylation, methylation, SUMOylation, and ISG15ylation among others) also regulate their function and response. PIAS1 is thought to interfere with STAT1 DNA binding and to recruit other transcriptional coregulators. PIAS proteins have also been shown to have E3 ligase activity and to promote protein SUMOylation. ${ }^{15}$ 
We identified 5 patients with disseminated dimorphic fungal infections who had mutationsin STAT1: 2 patients had disseminated refractory coccidioidomycosis beginning in childhood or adolescence without CMC, and 3 patients had disseminated histoplasmosis and CMC, including 1 patient who also had progressive multifocal leukoencephalopathy (PML). These are gain-of-function mutations that ultimately lead to delayed dephosphorylation of STAT1, lower STAT1 methylation, enhanced STAT1/PIAS1 association, and an impaired response to IFN- $\gamma$ restimulation.

\section{Methods}

\section{Patients and blood samples}

All samples were collected under approved National Institutes of Health (NIH) protocols; all patients or their parents provided written informed consent. Healthy volunteer blood samples were obtained under approved protocols through the Department of Transfusion Medicine, NIH.

\section{Cell lines}

EBV-transformed B-cell lines derived from patients and healthy donors were maintained in RPMI 1640 with 20\% FCS, $2 \mathrm{mmol} / \mathrm{L}$ L-glutamine, $100 \mathrm{U} / \mathrm{mL}$ penicillin, and $100 \mu \mathrm{g} / \mathrm{mL}$ streptomycin at $37^{\circ} \mathrm{C}$ in a humidified $5 \% \mathrm{CO}_{2}$ incubator. STAT1-deficient U3A cells (generously provided by G. Stark, Cleveland Clinic, Cleveland, Ohio) were maintained in complete Dulbecco modified Eagle medium (see the Methods section in this article's Online Repository at www.jacionline.org).

\section{STAT1 sequencing}

Genomic DNA (PureGene Gentra DNA isolation kit; Qiagen, Hilden, Germany) and total RNA (STAT-60 RNA isolation kit; Tel-Test, Friendswood, Tex) were extracted from EBVtransformed B-cell lines or polymorphonuclear leukocytes. Primers spanning exons and flanking splice sites of human genomic STAT1 and full-length cDNA were designed with Primer Select (Lasergene; DNASTAR, Madison, Wis). Genomic amplification was performed with Platinum PCR Supermix High Fidelity (Invitrogen, Carlsbad, Calif). Samples were treated with ExoSAP (Affymetrix, Santa Clara, Calif), and $1 \mu \mathrm{L}$ of the resulting product was used in sequencing reactions with Big Dye Terminators v3.1 (Applied Biosystems, Foster City, Calif), purified with Performa DTR short-well plate kit (Edge BioSystems, Gaithersburg, Md), and run on an Applied Biosystems 3730XL sequencer. Alignment was to the consensus sequence NM_007315.3 using Sequencer software (Gene Codes, Ann Arbor, Mich).

\section{Constructs}

Mutated STAT1 sequences or green fluorescent protein (GFP)-tagged constructs were created with a STAT1 expression vector (BioInnovatise, Rockville, Md). Wild-type (WT) and mutant STAT1 plasmids were isolated with the QIAprep Miniprep Kit (Qiagen), according to the manufacturer's recommendations, and all mutations were verified by means of sequencing. Transient transfection of U3A cells was done with the Nucleofactor Amaxa device (Lonza, Walkersville, Md), according to the manufacturer's recommendations.

\section{Reporter gene assay}

U3A cells were cotransfected with WT and/or mutant STAT1 expression constructs along with a plasmid containing tandem interferon-response elements (gamma-activated sequence [GAS] and type I interferon response element [ISRE]) driving a luciferase reporter gene (1 $\mu \mathrm{g}$; Panomics, Fremont, Calif). A Renilla expression vector was cotransfected to measure 
transfection efficiency. Cells were stimulated with human IFN- $\gamma$ or IFN-a2b at $1000 \mathrm{IU} / \mathrm{mL}$ for 6 hours. Luciferase activity was evaluated with a dual luciferase assay (Promega, Madison, Wis; see the Methods section in this article's Online Repository). Data are expressed as the fold increase in response to interferon over the WT unstimulated samples.

\section{Evaluation of STAT1 activation}

Phosphorylated STAT1 (pSTAT1) was assayed in U3A and EBV-B cells stimulated with IFN- $\gamma(400 \mathrm{IU} / \mathrm{mL})$ or IFN-a $(1,000 \mathrm{IU} / \mathrm{mL})$. For evaluation of dephosphorylation, pSTAT1 kinetics were assayed in cells stimulated with IFN- $\gamma$ from 30 to 120 minutes. Cell lysates were recovered and analyzed by means of Western blotting (WB) and flow cytometry. For immunoprecipitation, cell lysates were incubated with anti-STAT1 antibody and protein GSepharose (Amersham Biosciences, Piscataway, NJ) overnight at $4^{\circ} \mathrm{C}$, and immunoreactive proteins were resolved by means of WB.

\section{Downregulation of PIAS1}

High-purity small interfering RNA (siRNA) oligonucleotides that target PIAS1 and a control siRNA were obtained from Darmacon (Thermo Scientific, Lafayette, Colo). U3A cells were transiently transfected with the siRNA (ON-TARGETplus SMARTpool siRNA, $50 \mathrm{nmol} / \mathrm{L}$ ) through eletroporation (Nucleofactor Amaxa), cotransfected with WT or mutant STAT1 constructs, and stimulated with IFN- $\gamma(400 \mathrm{IU} / \mathrm{mL})$.

\section{Real-time PCR}

Total RNA was extracted from cultured cells (PBMCs isolated from venous blood by means of density centrifugation and transfected U3A cells) with the RNeasy Mini Kit (Qiagen). For real-time PCR, $1 \mu \mathrm{g}$ of total RNA was reverse transcribed (Invitrogen), and the resulting cDNA was amplified by means of PCR with the ABI 7500 Sequencer and TaqMan expression assays (Applied Biosystems). Glyceraldehyde-3-phosphate dehydrogenase was used as a normalization control. The data were analyzed with the $2^{-\Delta \Delta C T}$ method, and results were expressed as mean fold induction.

\section{Statistical analysis}

Results are reported as means \pm SDs, unless otherwise stated. Differences between groups were assessed by using the Student $t$ test (GraphPad Prism; GraphPad Software, San Diego, Calif). The statistical significance level adopted was a $P$ value of less than .05 .

\section{Results}

Patient 1 is a Hispanic female native of Arizona with no relevant previous or family history who presented at 14 years of age with extensive persistent tinea capitis and kerion caused by Trichophyton tonsurans. At age 17 years, she had prolonged cough, a painless right neck mass, fatigue, and weight loss. Computed tomography (CT) demonstrated multiple nodules throughout both lungs. CT confirmed diffuse progression with new osteomyelitis at vertebral bodies $\mathrm{C} 6$ through T5 and multiple lesions throughout the liver and spleen. She received liposomal amphotericin $\mathrm{B}(\mathrm{L}-\mathrm{AmB})$ and voriconazole and then posaconazole. At age 20 years, a new Coccidioides species-induced skin lesion developed that was associated with anorexia and weight loss. Magnetic resonance imaging (MRI) demonstrated progressive disease throughout (Fig 1, A), including a new intramedullary spinal cord lesion at T9 with cord edema (Fig 1, B).

Patient 2 is a white girl native to Arizona who presented at age 91/2 years with 3 weeks of nightly fevers and cough. She had multiple pulmonary nodules, massive necrotic intrathoracic lymphadenopathy with compression of the right mainstem bronchus and 
vasculature, and lesions of the manubrium and L4, L5, T8, and T9 vertebrae.

Coccidioidomycosis was diagnosed by means of serology, leading to fluconazole therapy. Fluconazole was changed to itraconazole, but the pulmonary and intrathoracic lesions continued to enlarge (see Fig E1 and the Results section in this article's Online Repository at www.jacionline.org). At age 131/2 years, multiple ring-enhancing lesions appeared in both cerebral hemispheres and the cerebellum. A right subretinal mass thought to be coccidioidal was treated with intraocular amphotericin B without improvement. Despite aggressive treatment with caspofungin, voriconazole, posaconazole, and steroids for inflammatory control, she died of overwhelming Coccidioides species infection at 17 years.

Patient 3 is a 21-year-old white man with a lifetime history of recurrent infections; 6 fractures in childhood, including the long and short bones; muscle weakness and atrophy; and bronchiectasis. Thrush appeared at 7 days of life and persisted for 4 years despite topical antifungal therapies. He also had onychomycosis. Fluconazole was started at age 4 years, and fungal infections resolved. At the same age, a single cervical node involved with Mycobacterium fortuitum was surgically excised. At age 12 years, he had severe disseminated histoplasmosis, which responded to itraconazole. Since age 15 years, he has had progressive bilateral upper limb muscle atrophy and weakness of unclear cause.

Patient 4 is a 31-year-old man who presented with disseminated histoplasmosis at age 17 years. He had tympanostomy tubes placed as a child without subsequent ear infections. He often had oral sores, and at age 16 years, he was hospitalized for oral candidiasis with probable esophageal involvement. At age 17 years, he had lymphadenopathy, fever, and weight loss caused by histoplasmosis involving the liver, bone marrow, and lymph nodes. At age 30 years, headaches and ataxia led to the discovery of multiple brain lesions.

Craniotomy and biopsy confirmed the largest lesion to be Histoplasma capsulatum. At age 31 years, brain biopsy demonstrated PML caused by JC virus detected on immunohistochemistry. During treatment with IL-2, he had Pseudomonas aeruginosainduced sepsis and died.

Patient 5 is a 25 -year-old woman born to unrelated parents who had disseminated histoplasmosis at age 7 years characterized by fever, hepatosplenomegaly, lymphadenopathy, and dyspnea. H capsulatum on lymph node and lung biopsies was successfully treated with itraconazole. At age 8 years, histoplasmosis in sputum associated with dyspnea and lymphadenopathy was successfully retreated with itraconazole. After completion of treatment, she had recurrent oral, cutaneous, and vaginal candidiasis. Neither her siblings nor her parents have fungal infections or autoimmunity.

\section{STAT1 mutations}

Full-length sequencing of STAT1 genomic and complementary DNA identified STAT1 heterozygous mutations in each patient. Patient 1 had c.1057G>A, E353K in the DNAbinding domain; patient 2 had c.800C $>\mathrm{T}$, A267V in the coiled-coiled domain ${ }^{11,12}$; patient 3 had c.1154C > T, T385M in the DNA-binding domain; and patient 4 had c.820C $>$ G, R274G and patient 5 had c.514T $>$ C, F172L, both of which were in the coiled-coil domain (Fig 2, A). $\mathrm{R} 274 \mathrm{G}$ is a different amino acid change at the same location as one previously reported in patients with CMC. ${ }^{12}$ In all cases sequencing of full-length cDNA demonstrated equal representation of mutant and WT alleles, indicating stability of the mutant mRNA. None of the patients' parents (patient 4's parents were not tested) carried the identified mutations, nor were these mutations found in dbSNP 132 or the 1000 Genomes Project. 


\section{Delayed STAT1 dephosphorylation, enhanced DNA binding, and transactivation}

STAT1-deficient U3A cells were transfected with WT and mutant STAT1 alleles to evaluate the activity of the STAT1 mutant proteins. Immunoblotting confirmed equal expression in transfected cells (Fig 2, B).

The STAT1 mutants in patients with histoplasmosis and coccidioidomycosis, as well as F172L (a critical site for STAT1 dephosphorylation), ${ }^{16}$ showed enhanced IFN- $\gamma-$ and IFNa-induced STAT1 phosphorylation in EBV-B cells (patients 1, 2, and 3: E353K, A267V, and T385M, respectively) and transfected U3As (R274G and F172L) compared with WT cells (see Fig E2, A, in this article's Online Repository at www.jacionline.org). Confocal microscopy (see the Results section in this article's Online Repository at Www.jacionline.org) confirmed the ability of STAT1 mutants to translocate to the nucleus after activation (Fig E2, B). These gain-of-function mutations led to persistent STAT1 phosphorylation for up to 120 minutes, a time at which healthy subjects had almost completely returned to baseline levels (Fig 3, A). Experiments with the kinase inhibitor staurosporine (see Fig E2, C) and flow cytometry confirmed the impaired dephosphorylation of the mutant proteins (Fig 3, B).

STAT1 GAS-binding activity in stimulated EBV-B cells from patients with gain-of-function mutants but not the dominant negative mutant L706S was enhanced in response to interferons when compared with that seen in healthy subjects (see Fig E3, A, in this article's Online Repository at www.jacionline.org). Assessment of transactivation response in U3A cells transfected with mutant constructs also showed enhanced activation of the GASluciferase reporter after IFN- $\gamma$ and IFN-a stimulation compared with WT cells (Fig 3, C). When WT STAT1 was cotransfected with the mutants, the enhanced activity remained essentially unchanged, confirming the dominant gain of function exerted by the mutant alleles (Fig 3, C). Interestingly, cotransfection of U3A cells with these STAT1 mutants along with the L706S dominant negative STAT1 construct showed the gain-of-function mutants to overcome the dominant negative mutant (Fig 3, D). The IFN-a-induced transcription activity in cells cotransfected with a dependent type I interferon response element (ISRE) was not different than that observed for the WT cells (see Fig E3, B).

\section{PIAS1-STAT1 interaction}

PIAS1 modulates STAT1 activity, and STAT1/PIAS1 interaction is also reported to be modulated by methylation of STAT1 ${ }^{17,18}$ After stimulation with interferons, PIAS1/STAT1 interaction was enhanced in patients' B cells compared with healthy donors when cell lysates were immunoprecipitated for STAT1 and blotted for associated PIAS1 (Fig 4, A). Moreover, immunoprecipitated lysates immunoblotted with a mono/dimethylarginine antibody (DMA) showed diminished methyl-STAT1 in stimulated mutant transfected U3A or EBV-B cells compared with normal cells (see Fig E4, A, in this article's Online Repository at www.jacionline.org). Treatment of cells with the well-characterized methyl donor S-adenosylmethionine (SAMe; 1600 nmol/L; Sigma-Aldrich, St Louis, Mo), which donates to the terminal nitrogen of arginine residues on target proteins, ${ }^{19}$ led to enhanced methyl-associated STAT1 (see Fig E4, B), decreased STAT1/PIAS1 association (Fig 4, B), and reduced IFN- $\gamma$-induced STAT1 phosphorylation compared with that seen in untreated cells (see Fig E4, C).

\section{Gene expression}

The effects of these novel dominant STAT1 mutations on IFN- gamma;-inducible target genes (CXC chemokine ligand 9 [CXCL9] and CXCL19 IP10], see Fig E5, A, in this article's Online Repository at www.jacionline.org) but not on traditional IFN-a target genes ( $M X 1$ and $I S G 15$, see Fig E5, B) were enhanced in U3A cells carrying mutant STAT1 
constructs compared with WT STAT1. To better understand the connection of STAT1 hyperactivation and impaired response in the IFN- $\gamma$ axis, we examined whether the hyperresponsiveness induced by these mutations impaired later IFN- $\gamma$ responses. Transfected U3A cells were stimulated with IFN- $\gamma$ for 3 hours, washed free of IFN- $\gamma$ (IFN$\gamma$ restimulation), and then restimulated with IFN- $\gamma$ for an additional 3 hours (IFN- $\gamma / \mathrm{IFN}-\gamma$ ). In these experiments WT STAT1-expressing cells showed clear-cut ability to augment gene expression (CXCL9 and $C X C L 10)$ after both stimulation and restimulation. In contrast, mutant $S T A T 1$ cells were able to upregulate their response to the initial IFN- $\gamma$ stimulation but not restimulation (Fig 5). These results were reproduced when using primary patients' PBMCs restimulated in vitro (see Fig E5, C). Failure to respond to restimulation with IFN- $\gamma$ was also observed for the mutations K286I and T288A, which have been described as being associated with CMC alone (see Fig E5, D). ${ }^{12}$

We investigated whether PIAS1 plays a role in the impaired gene expression seen after restimulation. Knockdown of PIAS1 (see Fig E6 in this article's Online Repository at www.jacionline.org) in U3A cells (but not control siRNA, data not shown) cotransfected with gain-of-function STAT1 mutants led to near normalization of gene expression after restimulation (Fig 6).

\section{Cytokine production and evaluation of $T_{H} 17$ response}

Secretion of proinflammatory cytokines (TNF- $a$ and IL-12p70) was upregulated in patients compared with healthy subjects. The $\mathrm{T}_{\mathrm{H}} 17$ response assayed in PBMCs

$\left(\mathrm{CD}^{+}{ }^{+} \mathrm{CD} 4{ }^{+} \mathrm{CD} 45 \mathrm{RO}^{+}\right.$cells) was low for the patients carrying the mutations $\mathrm{A} 267 \mathrm{~V}$, E353K, and T385M (see Fig E7 in this article's Online Repository at www.jacionline.org) but not in a patient missing IFN- $\gamma$ receptor $1(\operatorname{del} I F N G R I)$ or one with disseminated coccidioidomycosis without a recognized mutation, suggesting that defective production of $\mathrm{T}_{\mathrm{H}} 17$ cells is not necessary for the development of disseminated dimorphic fungal infection.

\section{Discussion}

Genetic defects in the IFN- $\gamma / \mathrm{IL}-12$ pathway have been found in patients with severe disseminated histoplasmosis and coccidioidomycosis, ${ }^{3-6}$ indicating that underlying deficiencies in the same genes that control nontuberculous mycobacteria and Salmonella species are also involved in the control of intracellular fungal disease, which was the reason to investigate this pathway in our patients in the first place.

Coccidioidomycosis is endemic in the American Southwest into South America, but most disease is limited and transient. However, dissemination occurs more often in certain ethnic groups, most notably African American and Filipino subjects, suggesting underlying genetic contributions. ${ }^{20,21}$ Both of our patients with disseminated coccidioidomycosis also had years of progressive pulmonary involvement without associated lung cavitation. Such chronic manifestations are so distinctly unusual for patients with coccidioidal infection that it might be specifically associated with aberrant STAT1 function. H capsulatum occurs worldwide, typically as a limited disease, but disseminated disease signifies immunodeficiency. ${ }^{22}$ Extrapulmonary coccidioidomycosis and histoplasmosis have also been described in patients with hyper-IgE syndrome caused by STAT3 mutations, ${ }^{23,24}$ even though infections are less severe.

The mutations we identified in STAT1 were in the coiled-coil and DNA-binding domains and led to severe fungal infections with or without CMC. Notably, patient 4 also had warts and PML. Recurrent oral herpes virus infections have been reported in STAT1 gain-offunction mutations, ${ }^{25}$ as well as severe herpes simplex virus and varicella zoster virus infections (Uzel et al, accompanying submission). The occurrence of viral, mycobacterial, 
and dimorphic fungal infections with these mutations in STAT1 suggests a functional overlap of the gain- and loss-of-function mutations, which can converge on the integrity of secondary responses to IFN- $\gamma$.

Members of the PIAS family negatively regulate the Janus kinase-STAT pathway and modulate nuclear factor $\kappa \mathrm{B}$ signaling and other pathways. ${ }^{26,27}$ PIAS1 interacts with the amino terminal domain of STAT1 (amino acids 1-191), ${ }^{28}$ where arginine 31 is located, the described site for STAT1 and STAT3 methylation. ${ }^{17,29,30}$ PIAS can also act as a SUMO E3 ligase, which might be relevant because STAT1 SUMOylation seems to modulate the transcriptional activity of target genes, the expression of PIAS1-sensitive genes, and the extent of responsiveness to IFN- $\gamma \cdot{ }^{31}$ Chromatin immune precipitation studies on PIAS1deficient macrophages and transcriptional analysis of cells treated with interferon found increased STAT1 binding to the promoters of PIAS1-sensitive genes ( $C X C L 9$ and $C X C L 10$ ) but not PIAS1-insensitive genes, indicating a select population of genes regulated by PIAS1. 27,32 Analogously, lysine methylation modifies STAT3-mediated responses. ${ }^{33}$ Disruption of K140 caused persistent STAT3 phosphorylation, differentially modulated IL-6-induced gene expression, and downregulation of a subset of genes after restimulation.

Similarly, the STAT1 mutations we identified did not normally upregulate gene expression after restimulation. Moreover, reduction of PIAS1 in the mutant cells restored the restimulation response to IFN- $\gamma$, as observed in WT cells. It is noteworthy that the defect is brought out predominantly by restimulation, suggesting that the occurrence of IFN- $\gamma$ tachyphylaxis might be central to the defect in this condition.

STAT1/PIAS1 association can be modulated by STAT1 methylation. ${ }^{17,18,34,35}$ Treatment of patients' cells with SAMe reduced STAT1/PIAS1 association and decreased STAT1 phosphorylation and restored the restimulation response to IFN- $\gamma$ in vitro. SAMe is a principal biologic methyl donor, the precursor for polyamine biosynthesis, described to overcome hepatitis virus-associated STAT1 hypomethylation. ${ }^{19,34-36}$ SAMe might have potential therapeutic uses in patients with STAT1 mutants.

STAT1 has a critical role in the control of fungal and other infections. Human susceptibility to coccidioidomycosis and histoplasmosis is apparently enhanced by both loss- and gain-offunction mutations in the IFN- $\gamma / \mathrm{IL}-12$ pathway, likely through mechanisms other than the impaired IL-17 response that predisposes patients to CMC.

\section{Supplementary Material}

Refer to Web version on PubMed Central for supplementary material.

\section{Acknowledgments}

Supported by the Division of Intramural Research, National Institute of Allergy and Infectious Diseases, National Institutes of Health, and funded in part with federal funds from the National Cancer Institute, National Institutes of Health, under contract no. HHSN261200800001E. The content of this publication does not necessarily reflect the views or policies of the Department of Health and Human Services nor does mention of trade names, commercial products, or organizations imply endorsement by the US Government.

We thank Ervand Kristosturyan and Lev Heller for technical assistance and Steven Becker (Biological Imaging Facility/RTB, NIAID/NIH) for assistance with the confocal experiments.

\section{References}

1. Al-Muhsen S, Casanova JL. The genetic heterogeneity of Mendelian susceptibility to mycobacterial diseases. J Allergy Clin Immunol. 2008; 122:1043-51. [PubMed: 19084105] 
2. Haverkamp MH, van Dissel JT, Holland SM. Human host genetic factors in non-tuberculous mycobacterial infection: lessons from single gene disorders affecting innate and adaptive immunity and lessons from molecular defects in interferon-gamma-dependent signaling. Microbes Infect. 2006; 8:1157-66. [PubMed: 16520075]

3. Zerbe CS, Holland SM. Disseminated histoplasmosis in persons with interferon-gamma receptor 1 deficiency. Clin Infect Dis. 2005; 41:e38-41. [PubMed: 16028145]

4. Moraes-Vasconcelos D, Grumach AS, Yamaguti A, Andrade ME, Fieschi C, de Beaucoudrey L, et al. Paracoccidioides brasiliensis disseminated disease in a patient with inherited deficiency in the beta1 subunit of the interleukin (IL)-12/IL-23 receptor. Clin Infect Dis. 2005; 41:e31-7. [PubMed: 16028144]

5. Vinh DC, Masannat F, Dzioba RB, Galgiani JN, Holland SM. Refractory disseminated coccidioidomycosis and mycobacteriosis in interferon-gamma receptor 1 deficiency. Clin Infect Dis. 2009; 49:e62-5. [PubMed: 19681704]

6. Vinh DC, Schwartz B, Hsu AP, Miranda DJ, Valdez PA, Fink D, et al. Interleukin-12 receptor $\beta 1$ deficiency predisposing to disseminated Coccidioidomycosis. Clin Infect Dis. 2011; 52:e99-102. [PubMed: 21258095]

7. Casanova JL, Holland SM, Notarangelo LD. Inborn errors of human JAKs and STATs. Immunity. 2012; 36:515-28. [PubMed: 22520845]

8. Dupuis S, Dargemont C, Fieschi C, Thomassin N, Rosenzweig S, Harris J, et al. Impairment of mycobacterial but not viral immunity by a germline human STAT1 mutation. Science. 2001; 293:300-3. [PubMed: 11452125]

9. Chapgier A, Wynn RF, Jouanguy E, Filipe-Santos O, Zhang S, Feinberg J, et al. Human complete Stat-1 deficiency is associated with defective type I and II IFN responses in vitro but immunity to some low virulence viruses in vivo. J Immunol. 2006; 176:5078-83. [PubMed: 16585605]

10. Averbuch D, Chapgier A, Boisson-Dupuis S, Casanova JL, Engelhard D. The clinical spectrum of patients with deficiency of signal transducer and activator of transcription-1. Pediatr Infect Dis J. 2011; 30:352-5. [PubMed: 20962705]

11. van de Veerdonk FL, Plantinga TS, Hoischen A, Smeekens SP, Joosten LA, Gilissen C, et al. STAT1 mutations in autosomal dominant chronic mucocutaneous candidiasis. N Engl J Med. 2011; 365:54-61. [PubMed: 21714643]

12. Liu L, Okada S, Kong XF, Kreins AY, Cypowyj S, Abhyankar A, et al. Gain-of-function human STAT1 mutations impair IL-17 immunity and underlie chronic mucocutaneous candidiasis. J Exp Med. 2011; 208:1635-48. [PubMed: 21727188]

13. Tamiya T, Kashiwagi I, Takahashi R, Yasukawwa H, Yoshimura A. Suppressors of cytokine signaling (SOCS) proteins and JAK/STAT pathways. Regulation of T cell inflammation by SOCS1 and SOCS3. Arterioscler Thromb Vasc Biol. 2011; 31:980-5. [PubMed: 21508344]

14. Shuai K. Regulation of cytokine signaling pathways by PIAS proteins. Cell Res. 2006; 16:196202. [PubMed: 16474434]

15. Palvimo JJ. PIAS proteins as regulators of small ubiquitin-related modifier (SUMO) modifications and transcription. Biochem Soc Trans. 2007; 35:1405-8. [PubMed: 18031232]

16. Zhong M, Henriksen MA, Takeuchi K, Schaefer O, Liu B, ten Hoeve J, et al. Implications of an antiparallel dimeric structure of nonphosphorylated STAT1 for the activation-inactivation cycle. Proc Natl Acad Sci USA. 2005; 102:3966-71. [PubMed: 15753310]

17. Mowen KA, Tang J, Zhu W, Schurter BW, Shuai K, Herschman HR, et al. Arginine methylation of STAT1 modulates IFNalpha/beta-induced transcription. Cell. 2001; 104:731-41. [PubMed: 11257227]

18. Zhu W, Mustelin T, David M. Arginine methylation of STAT1 regulates its dephosphorylation by T cell protein tyrosine phosphatase. J Biol Chem. 2002; 277:35787-90. [PubMed: 12171910]

19. Anstee QM, Day CP. S-adenosylmethionine (SAMe) therapy in liver disease: a review of current evidence and clinical utility. J Hepatol. 2012; 57:1097-109. [PubMed: 22659519]

20. Adam RD, Elliott SP, Taljanovicc MS. The spectrum and presentation of disseminated coccidioidomycosis. Am J Med. 2009; 122:770-7. [PubMed: 19635278] 
21. Hector RF, Rutherford GW, Tsang CA, Erhart LM, McCotter O, Anderson SM, et al. The public health impact of coccidioidomycosis in Arizona and California. Int J Environ Res Public Health. 2011; 8:1150-73. [PubMed: 21695034]

22. Hostoffer RW, Berger M, Clark HT, Schreiber JR. Disseminated Histoplasma capsulatum in a patient with hyper IgM immunodeficiency. Pediatrics. 1994; 94:234-6. [PubMed: 8036080]

23. Powers AE, Bender JM, Kumánovics A, Ampofo K, Augustine N, Pavia AT, et al. Coccidioides immitis meningitis in a patient with hyperimmunoglobulin $\mathrm{E}$ syndrome due to a novel mutation in signal transducer and activator of transcription. Pediatr Infect Dis J. 2009; 28:664-6. [PubMed: 19483664]

24. Vinh DC, Sugui JA, Hsu AP, Freeman AF, Holland SM. Invasive fungal disease in autosomaldominanthyper-IgE syndrome. J Allergy Clin Immunol. 2010; 125:1389-90. [PubMed: 20392475]

25. Tóth B, Méhes L, Taskó S, Szalai Z, Tulassay Z, Cypowyj S, et al. Herpes in STAT1 gain-offunction mutation [corrected]. Lancet. 2012; 379:2500. [PubMed: 22748593]

26. Liu B, Yang R, Wong KA, Getman C, Stein N, Teitell MA, et al. Negative regulation of NFkappaB signaling by PIAS1. Mol Cell Biol. 2005; 25:1113-23. [PubMed: 15657437]

27. Shuai K, Liu B. Regulation of gene activation pathways by PIAS proteins in the immune system. Nat Rev Immunol. 2005; 5:593-605. [PubMed: 16056253]

28. Shuai K. Modulation of STAT signaling by STAT-interacting proteins. Oncogene. 2000; 19:263844. [PubMed: 10851063]

29. Wolf SS. The protein arginine methyltransferase family: anupdate about function, new perspectives and the physiological roleinhumans. Cell Mol Life Sci. 2009; 66:2109-21. [PubMed: 19300908]

30. Iwasaki H, Kovacic JC, Olive M, Beers JK, Yoshimoto T, Crook MF, et al. Disruption of protein arginine N-methyltransferase 2 regulates leptin signaling and produces leanness in vivo through loss of STAT3 methylation. Circ Res. 2010; 107:992-1001. [PubMed: 20798359]

31. Begitt A, Droescher M, Knobeloch KP, Vinkemeier U. SUMO conjugation of STAT1 protects cells from hyperresponsiveness to IFN $\gamma$. Blood. 2011; 118:1002-7. [PubMed: 21636857]

32. Liu B, Mink S, Wong KA, Stein N, Getman C, Dempsey PW, et al. PIAS1 selectively inhibits interferon-inducible genes and is important in innate immunity. Nat Immunol. 2004; 5:891-8. [PubMed: 15311277]

33. Yang J, Huang J, Dasgupta M, Sears N, Miyagi M, Wang B, et al. Reversible methylation of promoter-bound STAT3 by histone-modifying enzymes. Proc Natl Acad Sci U S A. 2010; 107:21499-504. [PubMed: 21098664]

34. Li J, Chen F, Zheng M, Zhu H, Zhao D, Liu W, et al. Inhibition of STAT1 methylation is involved in the resistance of hepatitis B virus to interferon alpha. Antiviral Res. 2010; 85:463-9. [PubMed: 19857525]

35. Feld JJ, Modi AA, El-Diwany R, Rotman Y, Thomas E, Ahlenstiel G, et al. S-Adenosylmethionine improves early viral responses and interferon-stimulated gene induction in hepatitis $\mathrm{C}$ nonresponders. Gastroenterology. 2011; 140:830-9. [PubMed: 20854821]

36. Filipowicz M, Bernsmeier C, Terracciano L, Duong FH, Heim MH. S-adenosylmethionine and betaine improve early virological response in chronic hepatitis $\mathrm{C}$ patients with previous nonresponse. PLoS One. 2010; 5:e15492. [PubMed: 21079746]

\section{Abbreviations}

$\begin{array}{ll}\text { CMC } & \text { Chronic mucocutaneous candidiasis } \\ \text { CSF } & \text { Cerebrospinal fluid } \\ \text { CT } & \text { Computed tomography } \\ \text { DMA } & \text { Mono/dimethylarginine antibody } \\ \text { GAS0 } & \text { Gamma-activated sequence } \\ \text { GFP } & \text { Green fluorescent protein }\end{array}$




$\begin{array}{ll}\text { ISRE } & \text { Type I interferon response element } \\ \text { L-AmB } & \text { Liposomal amphotericin B } \\ \text { MRI } & \text { Magnetic resonance imaging } \\ \text { NIH } & \text { National Institutes of Health } \\ \text { PIAS } & \text { Protein inhibitor of activated STAT } \\ \text { PML } & \text { Progressive multifocal leukoencephalopathy } \\ \text { pSTAT1 } & \text { Phosphorylated STAT1 } \\ \text { SAMe } & \text { S-adenosylmethionine } \\ \text { siRNA } & \text { Small interfering RNA } \\ \text { STAT1 } & \text { Signal transducer and activator of transcription 1 } \\ \text { WB } & \text { Western blotting } \\ \text { WT } & \text { Wild-type }\end{array}$




\section{Clinical implications}

STAT1 gain-of-function mutations predispose to disseminated dimorphic fungal infections. Prolonged STAT1 phosphorylation, hypomethylation, and impaired dephosphorylation impair IFN- $\gamma$ restimulation, leading to apparent tachyphylaxis. 
A
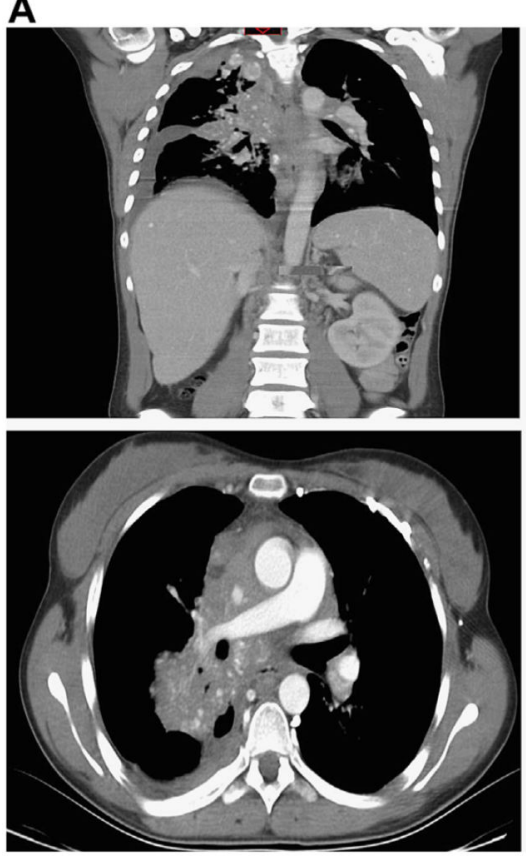

B

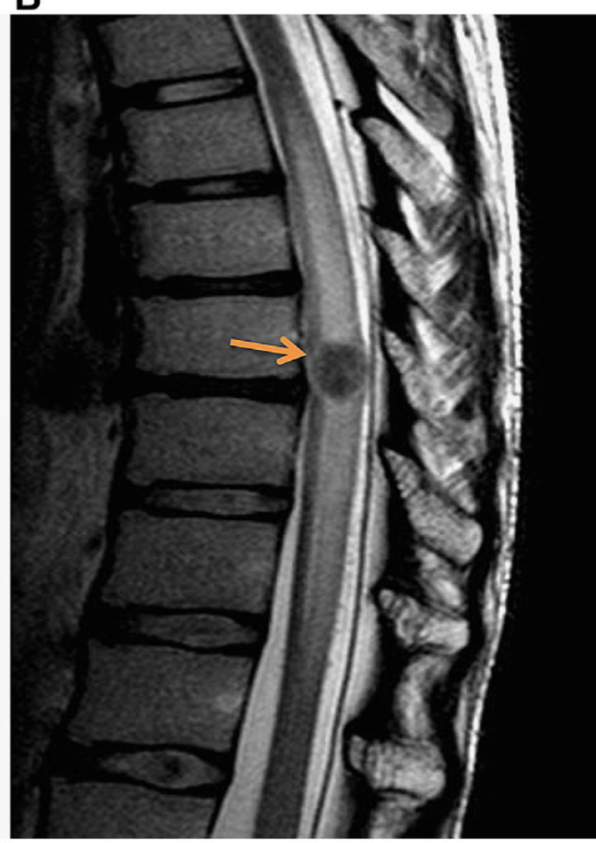

Fig 1.

Patient 1. A, Extensive right upper lung, mediastinal, and pleural involvement with $C$ immitis. B, MRI showing an intramedullary spinal cord lesion (arrow) with edema and cord compression. 
A

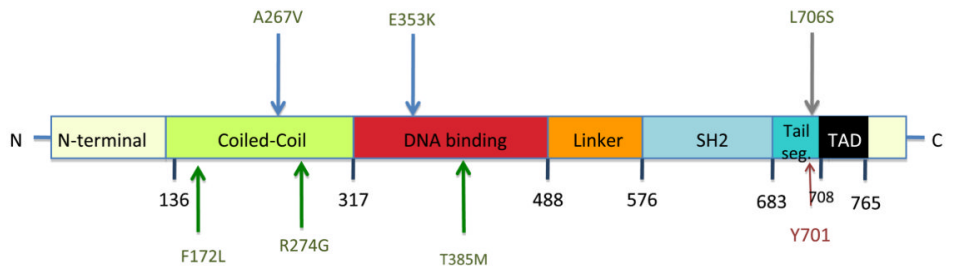

B

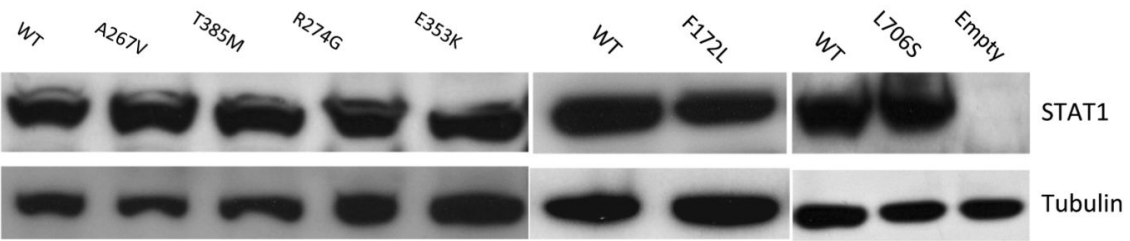

Fig 2.

STAT1 mutants. A, STAT1 coding region. TAD, Transactivation domain. Mutations were associated with (blue arrows) disseminated coccidioidomycosis and (green arrows) disseminated histoplasmosis. L706S was the dominant negative mutation. B, U3A cells transfected with STAT1 mutants, WT or empty vector, and WB with anti-STAT1 and antitubulin antibodies. 
A
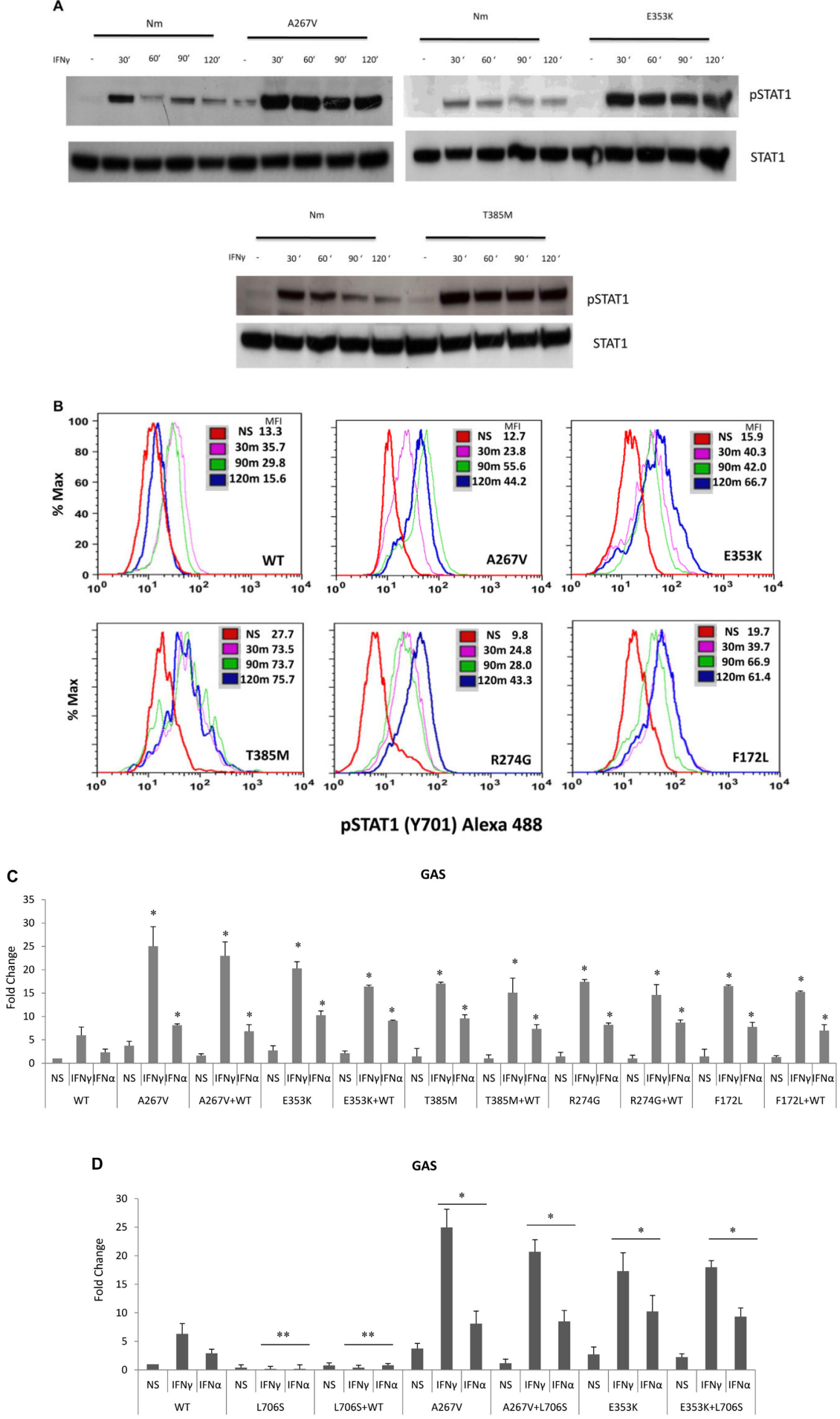

Fig 3.

STAT1 mutants lead to delayed dephosphorylation and enhanced luciferase GAS-induced activity. A, Dephosphorylation in EBV-B cells from patients and control subjects $(\mathrm{Nm})$ stimulated with IFN- $\gamma$ for the indicated periods. B, Dephosphorylation assayed by means of flow cytometry in U3A cells. Results are representative of at least 3 independent experiments. MFI, Mean fluorescence intensity; $N S$, nonstimulated. C, Transcriptional 
responses to IFN- $\gamma$ and IFN- $a$ in U3A cells transfected with STAT1 mutant constructs and when cotransfected with WT STAT1. D, U3A cells transfected with L706S showed no negative effect on the mutants A267V and E353K. Data show the mean fold increase relative to the WT nonstimulated specimens from a total of 5 experiments. $* P<.05$ when compared with stimulated WT, respectively. ${ }^{* *} P<.01$ compared with L706S. 
A
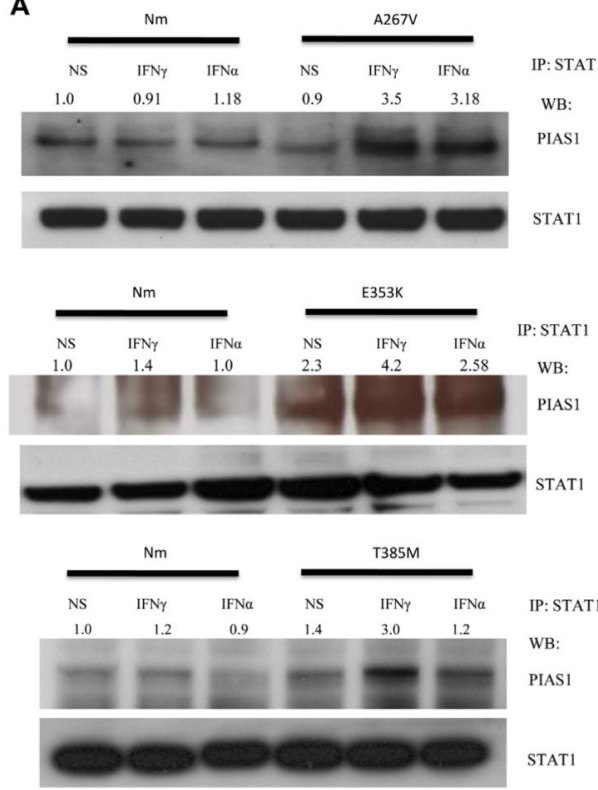

WB:

PIAS1

STAT1
B

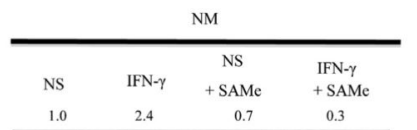

WB: STAT1
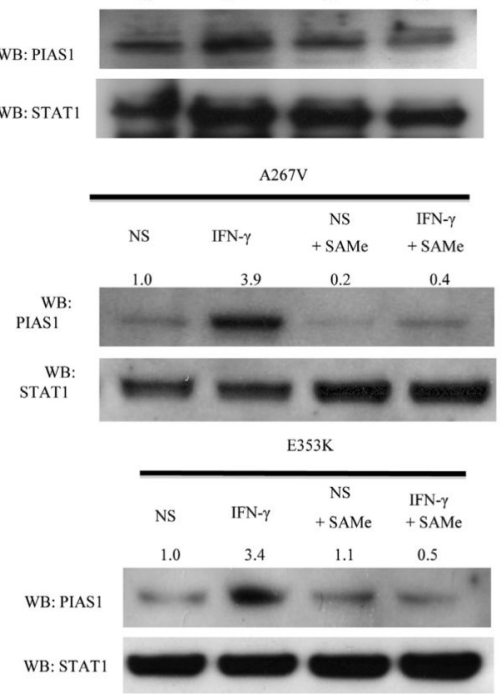

Fig 4.

Increased STAT1/PIAS1 association. A, After interferon stimulation, cell lysates from EBVB cells of patients and control subjects $(\mathrm{Nm})$ were IP:WB anti-STAT1: anti-PIAS1 antibody. B, EBV-B cells treated with SAMe were evaluated as above. Blots are representative of 3 independent experiments for each condition. Numbers are band densities normalized to the nonstimulated samples $(N S)$. 
A

\section{CXCL9}

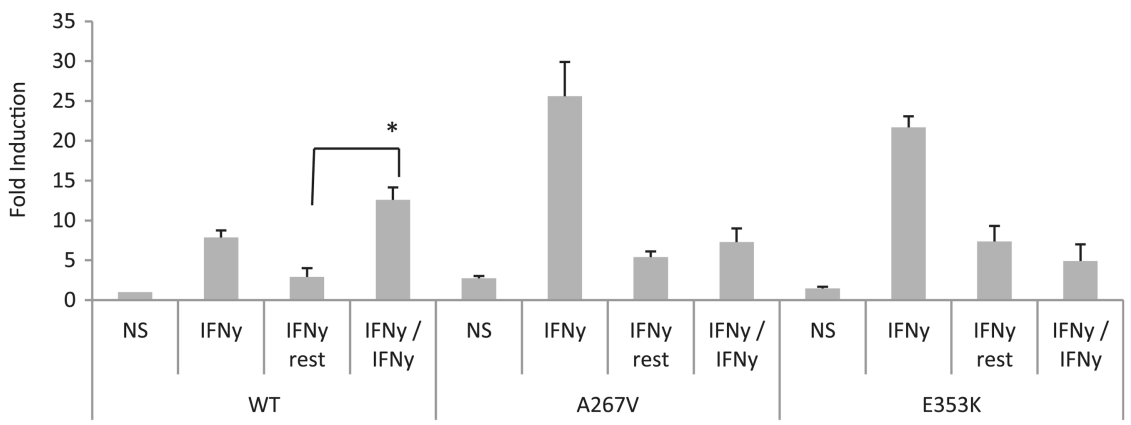

CXCL9

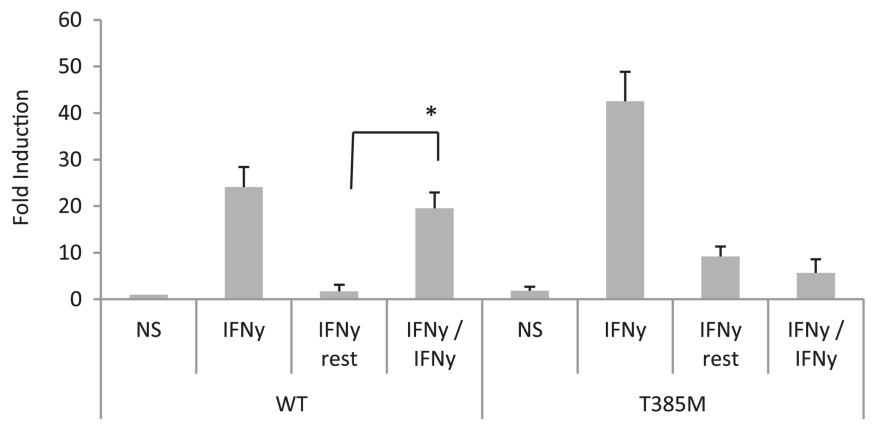

B

CXCL10
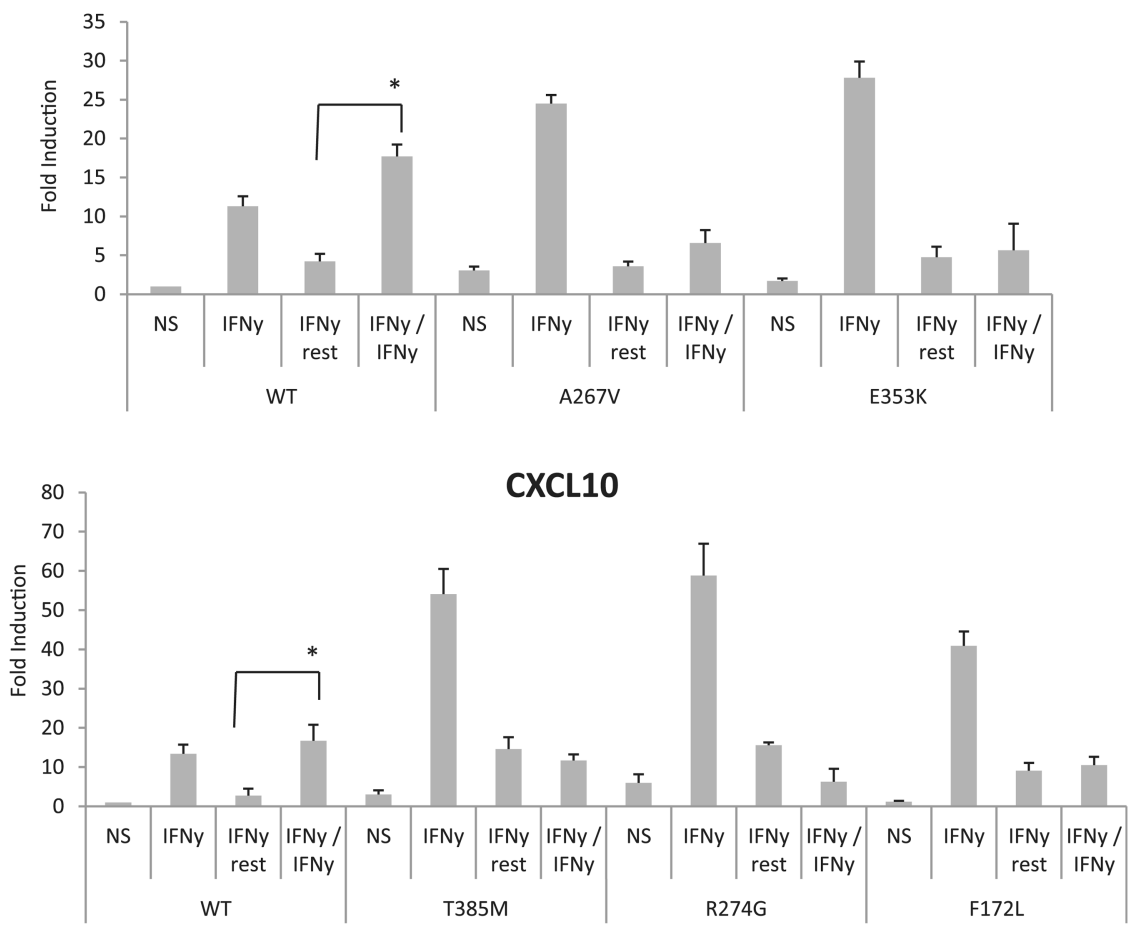

Fig 5.

Gene expression: restimulation experiments in transfected U3A cells. Cells were stimulated or not $(N S)$ with IFN- $\gamma$, washed, and restimulated (IFN- $\gamma / I F N-\gamma)$ or not (IFN- $\gamma$ rest). 
Expression of IFN- $\gamma$ (CXCL9 [A] and CXCL10 [B]) target genes was evaluated. Results are means \pm SDs of 3 independent experiments. ${ }^{*} \mathrm{P}<.05$ compared with IFN $-\gamma$ rest. 
A
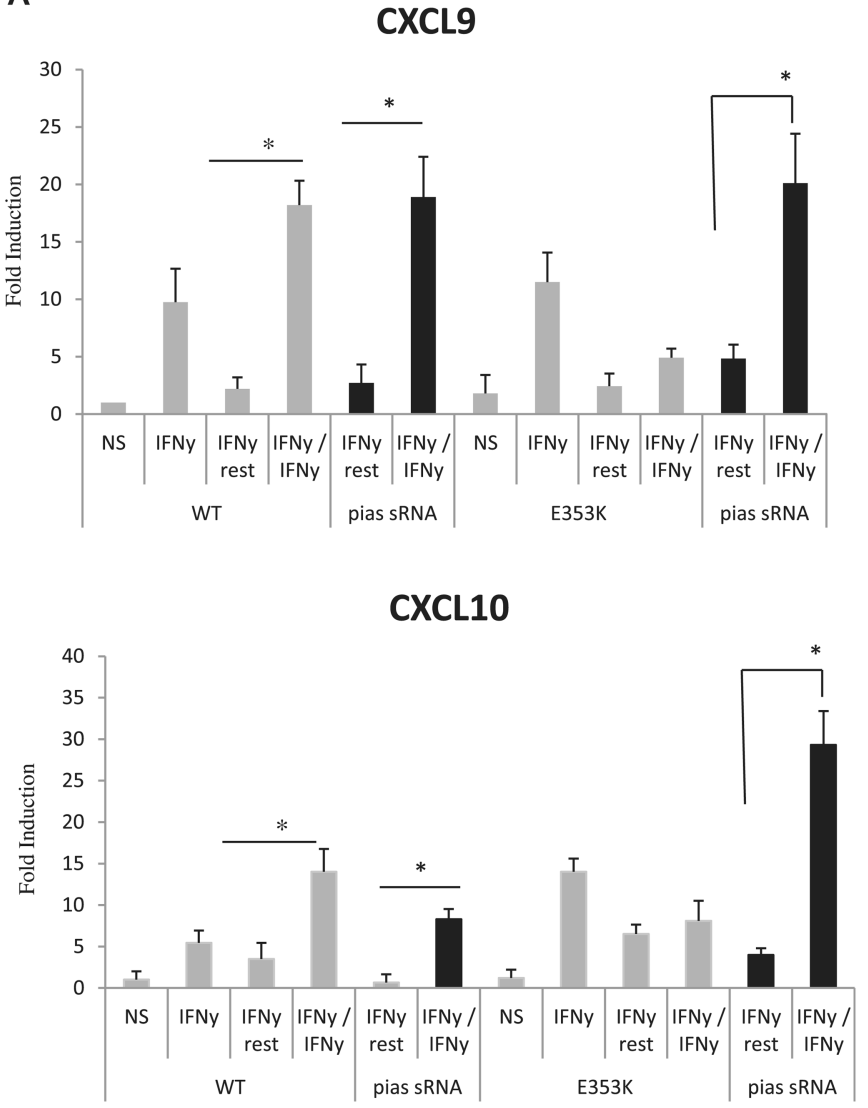

B

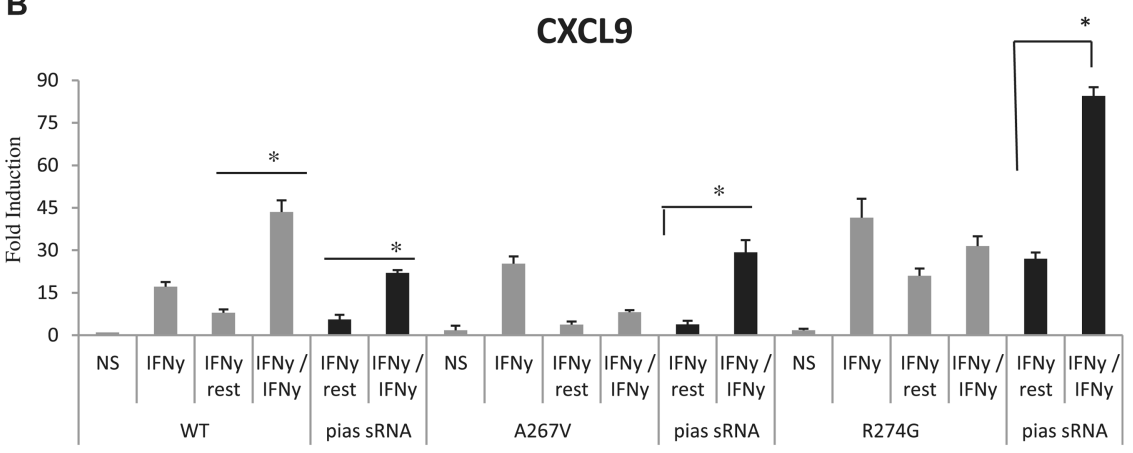

CXCL10

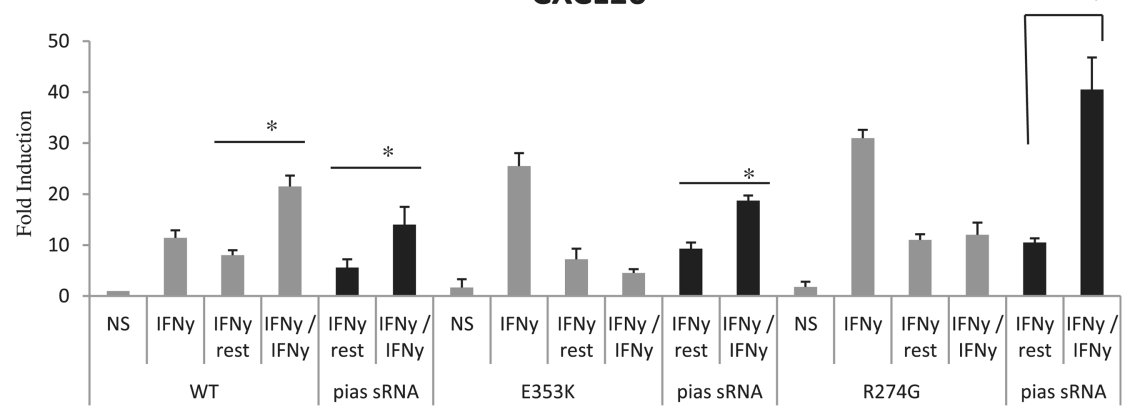

J Allergy Clin Immunol. Author manuscript; available in PMC 2014 June 01. 
Fig 6.

Reduction of PIAS1 modulates the IFN- $\gamma$-induced gene response. Evaluation of gene expression in U3A cells cotransfected with WT or mutant STAT1 and with siRNA directed against PIAS1. Cells were treated as described in Fig5. Restimulated cells $=$ IFN $-\gamma / \mathrm{IFN}-\gamma$ or PIAS siRNA IFN- $\gamma / \mathrm{IFN}-\gamma$. Results are means \pm SDs of 3 independent experiments. $N S$, Nonstimulated. $* P<.05$ when compared with IFN- $\gamma$ rest. A, WT and E353K with and without PIAS siRNA. B, WT, A267V, and R274G with and without PIAS siRNA. 